\title{
Research Letter \\ Reduction of Time-Dependent Schrödinger Equations with Effective Mass to Stationary Schrödinger Equations
}

\author{
Axel Schulze-Halberg \\ Department of Mathematics, University of Colima, Bernal Diaz del Castillo 340, Colima 28045, Mexico \\ Correspondence should be addressed to Axel Schulze-Halberg, xbat@ucol.mx
}

Received 22 October 2007; Accepted 18 November 2007

Recommended by R. Onofrio

We introduce a class of potentials for which the time-dependent Schrödinger equation with position-dependent (effective) mass allows reduction to a stationary Schrödinger equation. This reduction is done by a particular point canonical transformation which preserves $L^{2}$-normalizability.

Copyright (C) 2008 Axel Schulze-Halberg. This is an open access article distributed under the Creative Commons Attribution License, which permits unrestricted use, distribution, and reproduction in any medium, provided the original work is properly cited.

\section{INTRODUCTION}

Schrödinger equations with effective mass occur in the context of transport phenomena in crystals (e.g., semiconductors), where the electrons are not completely free, but interact with the potential of the lattice. The quantum dynamics of such electrons can be modeled by an effective mass, the behavior of which is determined by the band curvature [1-3]. Since the effective mass Schrödinger equation takes a more complicated form than the conventional Schrödinger equation [4], the identification of solvable cases is more difficult. In the stationary case, particular potentials with effective mass have been studied mainly by means of point canonical transformations [5-7] and Darboux transformations (resp., supersymmetric factorization) [8-10]. Recently, these methods have also been elaborated for the fully timedependent case [11-13]. However, the main problem of accessing time-dependent Schrödinger equations (TDSE) with effective mass is the lack of known solvable cases. In order to attack this problem for noneffective (constant) mass, it has been shown that for a certain class of potentials, the TDSE with constant mass can be mapped onto a stationary Schrödinger equation [14], such that each solvable stationary Schrödinger equation generates a solvable TDSE. Whereas in [14] the most general class of reducible potentials is derived, particular cases have been obtained earlier, for example, for time-dependent harmonic oscillator potentials with travelling-wave terms [15]. The method of mapping time-dependent onto stationary problems has also been used for the calculation of Green's functions for timedependent Coulomb and other potentials [16]. The purpose of the present note is to generalize the results in [14] to the effective mass case. We identify a class of potentials for which the effective mass TDSE can be reduced to a stationary Schrödinger equation by means of a point canonical transformation. Thus, each solvable stationary Schrödinger equation gives rise to a solvable effective mass TDSE. This allows the straightforward generation of time-dependent potentials with effective masses and their corresponding solutions. Furthermore, our transformation preserves $L^{2}$-normalizability, such that physical solutions are taken into physical solutions. In the remainder of this note, we first give the point canonical transformation that relates effective mass TDSEs and stationary Schrödinger equations to each other. We prove its correct simplification for constant mass and show that $L^{2}$-normalizability of the solutions is preserved. In the final paragraph we present an application.

\section{REDUCTION OF THE EFFECTIVE MASS TDSE}

\subsection{The point canonical transformation}

Let us consider the stationary Schrödinger equation

$$
\frac{1}{2 M} \Phi^{\prime \prime}+(E-U) \Phi=0
$$


where the mass $M$ is a positive constant, $U=U(x)$ denotes the potential with constant energy $E$, and $\Phi=\Phi(x)$ is the solution. Next, consider the effective mass TDSE

$$
i \Psi_{t}+\frac{1}{2 m} \Psi_{x x}-\frac{m_{x}}{2 m^{2}} \Psi_{x}-V \Psi=0,
$$

where $m=m(x, t)$ is the real-valued and positive mass, $V=V(x, t)$ stands for the potential, and $\Psi=\Psi(x, t)$ is the solution. Suppose that the potential $V$ in the effective mass TDSE can be expressed in the form

$$
\begin{aligned}
V= & U I_{3}^{2}+\frac{2 A B}{M^{3 / 2}} I_{1}-2\left(\frac{A}{M} I_{1}\right)^{2}-\frac{B}{2 \sqrt{M}} I_{2} \\
& -\frac{A}{M} I_{1} I_{2}-\frac{1}{8} I_{2}^{2}-\frac{7 m_{x}^{2}}{32 m^{3}}+\frac{m_{x x}}{8 m^{2}} \\
& +\int\left(\left(\frac{A \sqrt{m}}{M}+\frac{m_{t}}{4 \sqrt{m}}\right) I_{2}+\frac{\sqrt{m}}{2}\left(I_{2}\right)_{t}\right. \\
& +\left(\frac{2 A^{\prime} \sqrt{m}}{M}+\frac{A m_{t}}{M \sqrt{m}}\right) I_{1}+\frac{B^{\prime} \sqrt{m}}{\sqrt{M}} \\
& \left.+\frac{B m_{t}}{2 \sqrt{m M}}\right) d x+i\left(c^{\prime}-\frac{A}{M}\right)-\frac{B^{2}}{2 M}
\end{aligned}
$$

where the following abbreviations have been used:

$$
\begin{aligned}
& U=U\left(\sqrt{\left(\frac{1}{M}\right)} I_{3} I_{1}+\frac{1}{M} \int I_{3} B d t\right), \\
& I_{1}=\int \sqrt{m} d x, \quad I_{2}=\int \frac{m_{t}}{\sqrt{m}} d x, \\
& I_{3}=\exp \left(\frac{2}{M} \int A d t\right) .
\end{aligned}
$$

Then the solutions of the Schrödinger (1) and (2) are related to each other via the following point canonical transformation:

$$
\Psi(x, t)=\exp (f(x, t)-i E v(t)) \Phi(u(x, t)),
$$

where the functions $f, v$, and $u$ read as follows:

$$
\begin{aligned}
f=\int\left(\frac{m_{x}}{4 m}-i\left(\frac{\sqrt{m}}{2}\left(\int \frac{m_{t}}{\sqrt{m}} d x\right)\right.\right. \\
\left.\left.+\frac{2 A \sqrt{m}}{M}\left(\int \sqrt{m} d x\right)+\frac{B \sqrt{m}}{M}\right)\right) d x+c \\
u=\sqrt{\frac{1}{M}} \exp \left(\frac{2}{M} \int A d t\right)\left(\int \sqrt{m} d x\right) \\
+\frac{1}{M} \int \exp \left(\frac{2}{M} \int^{t} A d t^{\prime}\right) B d t \\
v=\int \exp \left(\frac{4}{M} \int A d t\right) d t
\end{aligned}
$$

with arbitrary, real-valued $A=A(t), B=B(t)$, and $c=c(t)$. The latter statement is proved in a straightforward manner by substitution of (6) with (7)-(9) into the effective mass TDSE (2). Thus, each effective mass TDSE with a potential of the form (3) can be reduced to a stationary Schrödinger equation by means of the point canonical transformation (6). Hence, if the stationary Schrödinger (1) is solvable, so is its effective mass counterpart (2).

\subsection{The constant mass case}

It has been shown that the TDSE with constant mass allows reduction to a stationary Schrödinger equation, if its potential takes a certain form [14]. Let us now verify that our potential simplifies correctly to this form if we assume the mass $m$ to be a constant. Thus, suppose that $m=M$ is constant and note that $m_{x}=m_{t}=0$. From (5), we then infer that $I_{1}=\sqrt{M} x$ and $I_{2}=0$. Now, it is easy to see that the potential (3) for constant $m=M$ takes the form

$$
\begin{aligned}
V= & U I_{3}^{2}+\left(A^{\prime}-\frac{2 A^{2}}{M}\right) x^{2}+\left(B^{\prime}-\frac{2 A B}{M}\right) x \\
& -\frac{B^{2}}{2 M}+i\left(c^{\prime}-\frac{A}{M}\right)
\end{aligned}
$$

which coincides with the potential given in [14].

\subsection{Preservation of $L^{2}$-normalizability}

In this paragraph, we show that the mapping (6) preserves $L^{2}$-normalizability. To this end, we assume that the solution $\Psi$ of the effective mass TDSE (2) has domain $D \subset \mathbb{R}$, and infer from (6) and (7) that

$$
\begin{aligned}
\|\Psi\|^{2} & =\int_{D}|\exp (f-i E v) \Phi|^{2} d x \\
& =\int_{D} \exp \left(\int^{x} \frac{m_{x^{\prime}}}{2 m} d x^{\prime}\right)|\Phi|^{2} d x
\end{aligned}
$$

Suppose now that the ratio $m_{x} / m$ is not constant or purely time-dependent. We change the integration variable from $x$ to $u$, where the latter is given in (8):

$$
\begin{aligned}
\int_{D} \exp & \left(\int^{x} \frac{m_{x^{\prime}}}{2 m} d x^{\prime}\right)|\Phi|^{2} d x \\
& =\int_{u(D)} \sqrt{m}|\Phi|^{2} \frac{\sqrt{M}}{I_{3} \sqrt{m}} d u=\int_{u(D)} \sqrt{m}|\Phi|^{2} \frac{1}{u^{\prime}(x)} d u \\
& =\frac{\sqrt{M}}{I_{3}}\|\Phi\|^{2}
\end{aligned}
$$

Thus, if $\Phi$ is $L^{2}$-normalizable on $u(D)$, then $\Psi$ is $L^{2}$ normalizable on $D$. Now, if the ratio $m_{x} / m$ is constant or purely time-dependent, the integral evaluation in (12) is invalid. Note that a constant ratio $m_{x} / m$ implies that the mass has the form of an exponential, that is,

$$
m=a \exp (b x)
$$


where $a$ and $b$ are purely time-dependent or constants. Insertion of the latter mass into (12) gives

$$
\begin{aligned}
\int_{D} \exp & \left(\int^{x} \frac{m_{x^{\prime}}}{2 m} d x^{\prime}\right)|\Phi|^{2} d x \\
& =\int_{u(D)} \exp \left(\frac{b}{2} x\right)|\Phi|^{2} \frac{1}{u^{\prime}(x)} d u \\
& =\int_{u(D)} \exp \left(\frac{b}{2} x\right)|\Phi|^{2} \frac{\sqrt{M}}{I_{3} \sqrt{m}} d u \\
& =\int_{u(D)} \exp \left(\frac{b}{2} x\right)|\Phi|^{2} \frac{\sqrt{M}}{I_{3} \sqrt{a} \exp ((b / 2) x)} d u \\
& =\frac{\sqrt{M}}{I_{3} \sqrt{a}}\|\Phi\|^{2} .
\end{aligned}
$$

As in (13), if $\Phi$ is $L^{2}$-normalizable on $u(D)$, then $\Psi$ is $L^{2}$ normalizable on $D$.

\subsection{Application}

We now apply the scheme developed above as follows. First, we choose a potential $U$, such that the associated stationary Schrödinger equation (1) is exactly solvable, and calculate its solution. In the second step, we choose an effective mass $m$ and fix the free parameters $A, B, c$ that appear in (3). By means of this data, we compute the transformation components $f, u, v$ that are defined in (7)-(9). In the final step, we obtain the time-dependent potential (3) and its solution (6). Thus, let us start with the stationary potential $U$, which we choose to be a harmonic oscillator:

$$
U=x^{2}
$$

The associated stationary Schrödinger equation (1) has the following solutions that are $L^{2}$-normalizable on any subset of $\mathbb{R}$ :

$$
\Phi_{n}=\exp \left(-\sqrt{\frac{M}{2}} x^{2}\right) H_{n}\left((2 M)^{1 / 4} x\right) .
$$

Here, $H_{n}$ denotes the Hermite polynomial of order $n$, where $n$ is a natural number or zero [17]. The corresponding energies read

$$
E_{n}=\sqrt{\frac{1}{2 M}}(2 n+1)
$$

Now let $\alpha=\alpha(t)$ be an arbitrary, real, and positive function and set

$$
\begin{aligned}
& m=\alpha \exp (x), \quad A=-\frac{M \alpha^{\prime}}{4 \alpha}, \\
& B=0, \quad c=0 .
\end{aligned}
$$

On substituting the latter settings into (7)-(9), we get

$$
\begin{aligned}
& f=\frac{1}{4} x, \quad u=\frac{2}{\sqrt{M}} \exp \left(\frac{1}{2} x\right), \\
& v=\int \frac{1}{\alpha} d t .
\end{aligned}
$$

We now insert (16) and (19) into the time-dependent potential (3), which simplifies to

$$
V=\frac{4}{M \alpha} \exp (x)-\frac{3}{32 \alpha} \exp (-x)
$$

Thus, the solution of the time-dependent effective mass Schrödinger equation (2) with exponential mass given in (19) and potential (21) can be obtained by insertion of (17), (18), and (20) into (6):

$$
\begin{aligned}
\Psi_{n}= & \exp \left(-\sqrt{\frac{8}{M}} \exp (x)+\frac{1}{4} x-i \frac{(2 n+1)}{\sqrt{2 M}} \int \frac{1}{\alpha} d t\right) \\
& \times H_{n}\left(\left(\frac{32}{M}\right)^{1 / 4} \exp \left(\frac{1}{2} x\right)\right) .
\end{aligned}
$$

Let us now exemplarily verify that the solution as given in (22) is $L^{2}$-normalizable. For the sake of simplicity, we restrict ourselves to the case $n=0$ and $M=1 / 2$. It is well known that the functions $\Phi_{n}$ as given in (17) are $L^{2}$-normalizable on the whole real line. In this application, we will restrict the domain of $\Phi_{n}$ to $(0, \infty)$, which preserves normalizability of $\Phi_{n}$. Note that now a possible boundary condition at zero has to be taken into account, as the energy spectrum (18) depends on it. From the definition of the Hermite polynomials [17], it follows immediately that

$$
\Phi_{n}(0)\left\{\begin{array}{l}
> \\
= \\
<
\end{array}\right\} 0 \quad \text { if } n=\left\{\begin{array}{c}
4 k \\
2 k+1 \\
4 k+2
\end{array}\right\}, \quad k \in \mathbb{N} \cup\{0\} .
$$

Thus, depending on the boundary condition imposed at zero, some values of the spectrum (18) must be omitted. In the present application, we do not require boundary conditions, since we focus on the transformation (20). Hence, the functions $\Phi_{n}$ are $L^{2}$-normalizable on $(0, \infty)$ and we find for $\Phi_{0}$ that

$$
\left\|\Phi_{0}\right\|^{2}=\int_{0}^{\infty} \exp \left(-x^{2}\right) d x=\frac{1}{2} \sqrt{\pi} .
$$

Before we can compute the norm of the solution $\Psi$ as given in (22), according to (11), we need to find the domain $D$. Since $(0, \infty)=u(D)$, by means of $u$ in $(20)$, we must have $D=\mathbb{R}$. We find

$$
\left\|\Psi_{0}\right\|^{2}=\int_{-\infty}^{\infty} \exp \left(-8 \exp (x)+\frac{1}{2} x\right) d x=\sqrt{\frac{\pi}{8}} .
$$

On comparing (24) and (25), we get

$$
\left\|\Psi_{0}\right\|^{2}=\sqrt{\frac{1}{2}}\left\|\Phi_{0}\right\|^{2} .
$$

We compare this with (15). According to (5), for $M=1 / 2$, we have $I_{3}=\sqrt{1 / \alpha}$, and according to (14), we have $a=\alpha$, which gives

$$
\frac{\sqrt{M}}{I_{3 \sqrt{a}}}=\sqrt{\frac{1}{2}}
$$

coinciding with the factor in (26). 


\section{CONCLUDING REMARKS}

The point canonical transformation (6)-(9) introduced in this note provides a method for generating solvable timedependent potentials for effective mass TDSEs. On one hand, such potentials are interesting in themselves, on the other hand, they can be used to find more potentials of their kind, for example, by looking for their supersymmetric partner potentials.

\section{REFERENCES}

[1] T. Gora and F. Williams, "Electronic states of homogeneous and inhomogeneous mixed semiconductors," in II-VI Semiconducting Compounds, D. G. Thomas, Ed., Benjamin, New York, NY, USA, 1967.

[2] T. Gora and F. Williams, "Theory of electronic states and transport in graded mixed semiconductors," Physical Review, vol. 177, no. 3, pp. 1179-1182, 1969.

[3] G. T. Landsberg, Solid State Theory: Methods and Applications, Wiley-Interscience, London, UK, 1969.

[4] K. C. Yung and J. H. Yee, "Derivation of the modified Schrödinger equation for a particle with a spatially varying mass through path integrals," Physical Review A, vol. 50, no. 1, pp. 104-106, 1994.

[5] A. de Souza Dutra and C. A. S. Almeida, "Exact solvability of potentials with spatially dependent effective masses," Physics Letters A, vol. 275, no. 1-2, pp. 25-30, 2000.

[6] O. Mustafa and S. H. Mazharimousavi, " $d$-dimensional generalization of the point canonical transformation for a quantum particle with position-dependent mass," Journal of Physics A, vol. 39, no. 33, pp. 10537-10547, 2006.

[7] C. Tezcan and R. Sever, "Exact solutions of the schrödinger equation with position-dependent effective mass via general point canonical transformation," Journal of Mathematical Chemistry, vol. 42, no. 3, pp. 387-395, 2007.

[8] A. Ganguly and L. M. Nieto, "Shape-invariant quantum Hamiltonian with position-dependent effective mass through second-order supersymmetry," Journal of Physics A, vol. 40, no. 26, pp. 7265-7281, 2007.

[9] A. Schulze-Halberg, "Darboux transformations for $\mathrm{N}$ dimensional effective mass schrödinger equations with hyperspherical symmetry," International Journal of Modern Physics A, vol. 22, no. 19, pp. 3293-3304, 2007.

[10] T. Tanaka, “ $\mathcal{N}$-fold supersymmetry in quantum systems with position-dependent mass," Journal of Physics A, vol. 39, no. 1, pp. 219-234, 2006.

[11] A. Schulze-Halberg, "Quantum systems with effective and time-dependent masses: form-preserving transformations and reality conditions," Central European Journal of Physics, vol. 3, no. 4, pp. 591-609, 2005.

[12] A. Schulze-Halberg, "Darboux transformations for timedependent schrödinger equations with effective mass," International Journal of Modern Physics A, vol. 21, no. 6, pp. 1359 $1377,2006$.

[13] A. Schulze-Halberg, "Supersymmetry of time-dependent Schrödinger equations with effective mass," to appear in Central European Journal of Physics.

[14] F. Finkel, A. González-López, N. Kamran, and M. A. Rodríguez, "On form-preserving transformations for the time-dependent Schrödinger equation," Journal of Mathematical Physics, vol. 40, no. 7, pp. 3268-3274, 1999.
[15] J. R. Ray, "Exact solutions to the time-dependent Schrödinger equation," Physical Review A, vol. 26, no. 2, pp. 729-733, 1982.

[16] V. V. Dodonov, V. I. Man'ko, and D. E. Nikonov, "Exact propagators for time-dependent Coulomb, delta and other potentials," Physics Letters A, vol. 162, no. 5, pp. 359-364, 1992.

[17] I. S. Gradshteyn and I. M. Ryzhik, Tables of Series, Products and Integrals, Academic Press, San Diego, Calif, USA, 1994. 

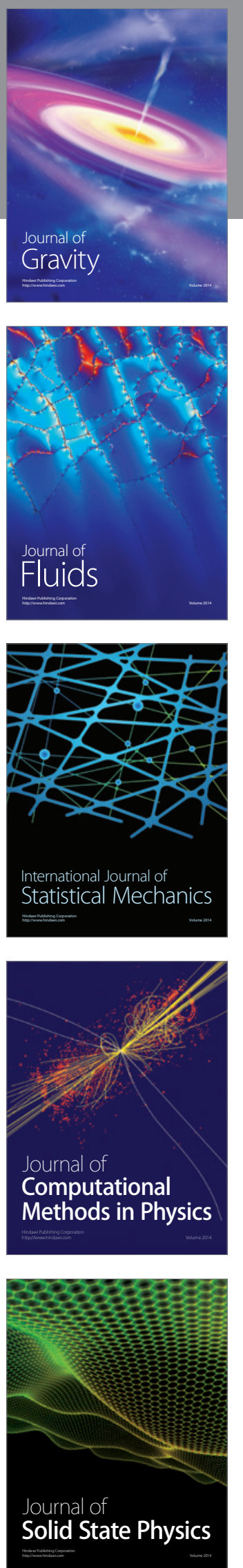

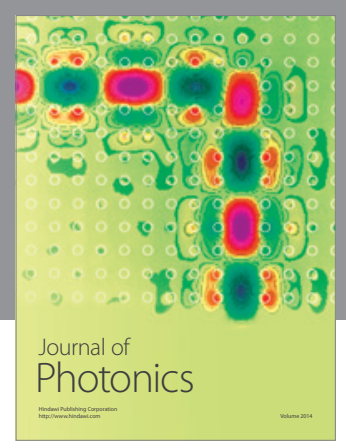

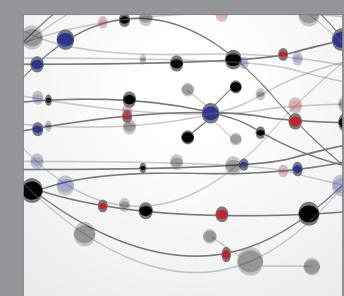

The Scientific World Journal
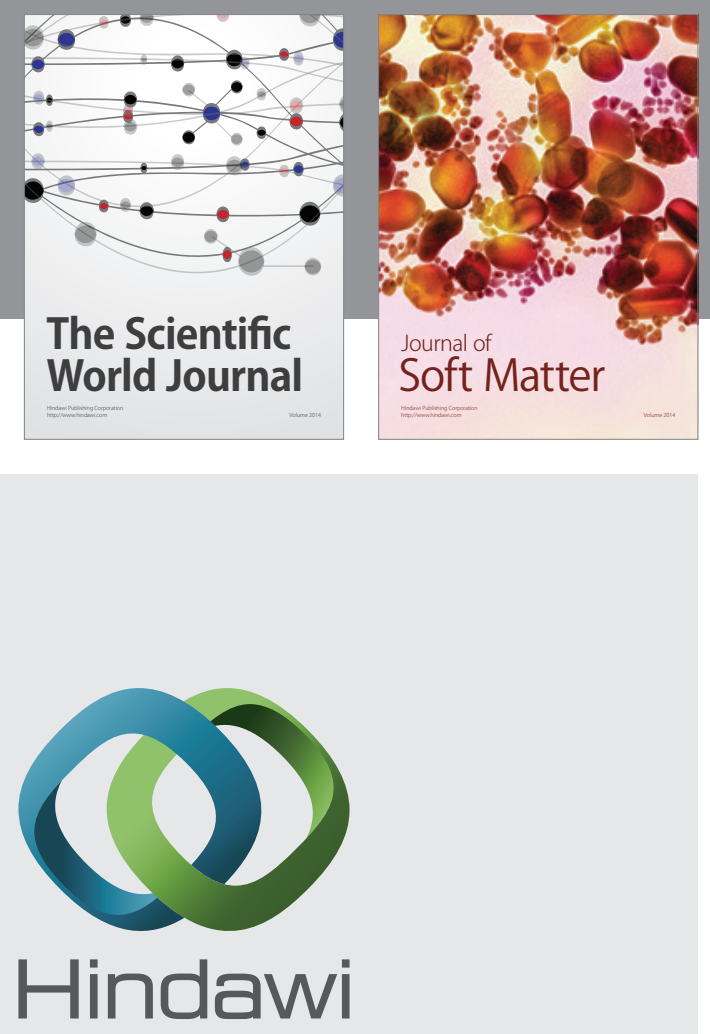

Submit your manuscripts at

http://www.hindawi.com
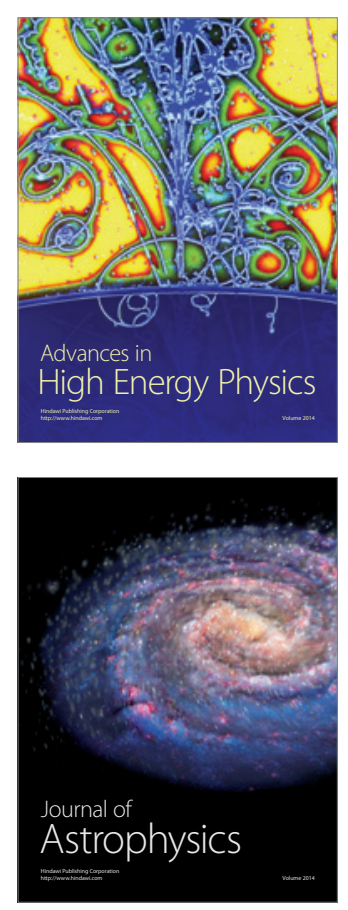
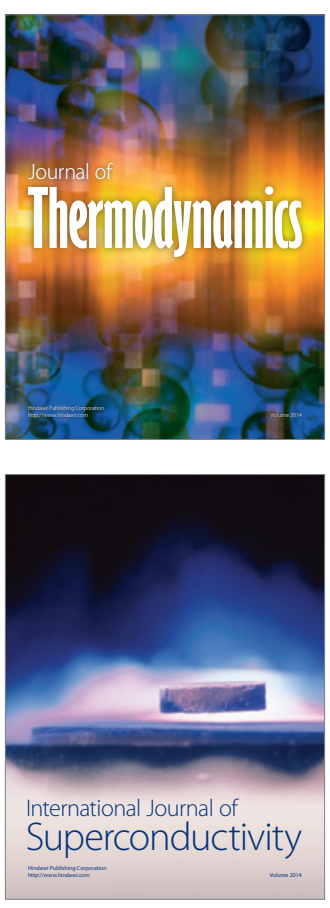
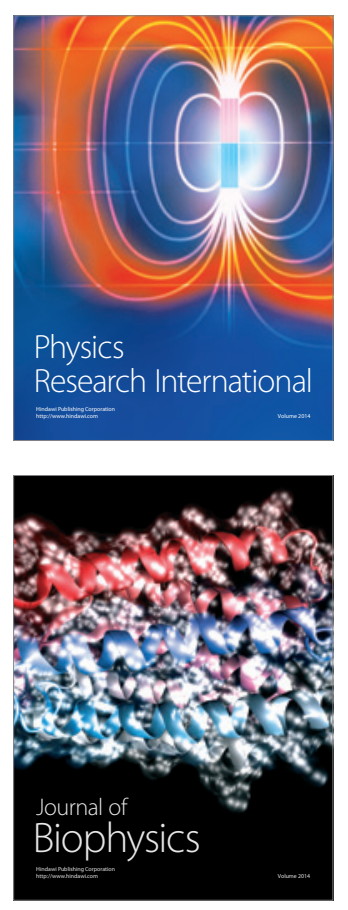
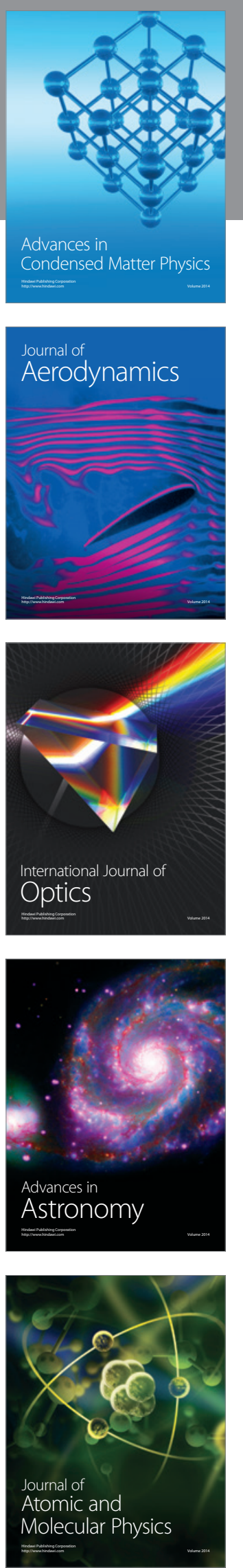